\title{
NARRATIVE LANDSCHAFTEN ALS LERNRAUM IM WANDEL
}

\begin{abstract}
Landschaften als Lerngegenstand und Lernort erschließen sich in der physischen Begegnung mit den materiellen, naturräumlichen Gegebenheiten und Artefakten im Zusammenwirken mit Immateriellem, wie etwa überlieferter Bau- und Entstehungsgeschichte oder auch lokal verankerten Geschichten im weiteren Sinne. Geschichten, von der kosmologischen Legende bis hin zur Alltagsanekdote, spiegeln oder interpretieren vorhandene Landschaftsformen und -elemente, können aber auch umgekehrt künstlerisch in eine Gestaltung des Raumes umgesetzt werden. Im explorativen Lernen in Kulturlandschaften, wie etwa in historischen Gärten und Parkanlagen, eröffnet die Auseinandersetzung mit der immateriellen Landschaft des Narrativen (»Storyscape«) vielfältige Zugänge, den Ort über historische und naturwissenschaftliche Daten hinaus als ein lebendes, erlebbares und erlebtes Denkmal zu erfahren. Dabei ist eine Landschaft, in der der Klimawandel in seinen Auswirkungen sichtbar wird, Quelle und Projektionsfläche neuer Narrative, die die Erfahrung des Raumes neu überformen.
\end{abstract}

Landscapes as subjects and sites of learning can be understood through their physical encounter with the tangible natural space realities and artefacts in interaction with intangible elements such as, for instance, handed down architectural and development history or also locally anchored narratives in a wider sense. Stories ranging from the cosmological legend down to everyday anecdotes reflect or interpret existing landscape forms and components but can also conversely be incorporated artistically into the design of the space. In explorative learning in cultivated landscapes, such as historical gardens and parks, critical examination of the intangible storyscape opens up diverse ways of experiencing the site, beyond the historical and natural scientific data, as a living, historical monument that can

Im Text werden Maskulinum und Femininum verwendet, wenn es um Personen geht. Gemeint sind grundsätzlich alle Menschen, gleich welcher Geschlechtsidentität sie sich zugehörig fühlen. 
be experienced. In this context a landscape in which the effects of climate change are visible as both the source and the projection site for new narratives which reshape the experience of the space.

\section{Geschützte Landschaften als Lernraum}

Eine Landschaft besteht aus diskreten Objekten und entwickelt sich in kontinuierlichen, manchmal auch plötzlich und unvorhersehbar ausgelösten Prozessen (Tang 2008, 59; Antrop/Van Eetvelde 2017, 91). Dem Menschen kommen »Produkte« und »Leistungen «, die aus diesem dynamischen Gefüge erwachsen, zugute. Über die offenkundigen materiellen Ressourcen wie landwirtschaftliche Nutzflächen, Wälder, Weiden und Gewässer hinaus ist es häufig Immaterielles, das für eine besondere Region bezeichnend ist und ihr einen spezifischen Charakter, Bedeutung, >Atmosphäre $<$ und einen besonderen >Wert gibt (Watson 1951). ${ }^{1}$ "Immaterielle Leistungen " sind etwa die zur Betrachtung einladende landschaftliche Schönheit, der in der Betrachtung erwachsende Erkenntnisgewinn oder die erhebenden Gefühle, auf den Spuren früherer Kulturen zu wandeln, die Reisende des 19. Jahrhunderts auf der Grand Tour in Italien oder »das Land der Griechen mit der Seele suchend « empfanden.

Der Schutz von Objekten und Prozessen zur Erhaltung der Landschaft ist erforderlich, damit Leistungen wie diese, oft auf immaterielle Aspekte bezogene ästhetische Bildungsfunktion nachhaltig erbracht werden kann. Die Bedeutung geschützter Landschaftsausschnitte als Bildungsraum und ihre Funktion der »Bewusstseinsbildung und Kommunikation zur biologischen Vielfalt« (Bundesamt für Naturschutz 2010) wurde vielfach betont. Aufgaben der »naturkundlichen Bildung « kommen beispielsweise den Nationalparks zu ( $\$ 24$ (2) BNatSchG) und auch Biosphärenreservate dienen explizit der »Bildung für nachhaltige Entwicklung «, der »Forschung « und der »Beobachtung von Natur und Landschaft» $(\$ 25$ (2) BNatSchG). Es sind Orte, an denen »[n]eben dem Schutz der Arten- und Biotopvielfalt $[. .$.$] auch umweltgerechte Wirtschaftsweisen entwickelt und erprobt werden « und$ so »diese Gebiete als Modellregionen für eine nachhaltige Entwicklung dienen« (Bundesamt für Naturschutz 2010; $\$ 25, \mathrm{BNatSchG).}$

Internationale Aufmerksamkeit genießen solche geschützten Flächen, die als UNESCOWeltkultur- oder Naturerbe ausgezeichnet sind, die infolgedessen auch in erhöhtem Maße Ziel touristischer Aktivitäten sind und denen daher auch ein besonderes Potential für verschiedenste Bildungsaktivitäten innewohnt. Die `Hildesheimer Resolution` (2006) der Deutschen UNESCO-Kommission spricht explizit vom »Bildungsauftrag der deutschen Welterbestätten « (Deutsche UNESCO-Kommission 2006; Ströter-Bender 2011, 35). Die >Lübecker Erklärung`(2017) betont die Rolle der Welterbestätten »als Stätten der Bildung,

1 Vgl. die Beiträge von Florian Abe und Karen David in diesem Band. 
an denen Kinder und Jugendliche den interkulturellen Zugang zur eigenen Geschichte, zur Geschichte anderer Völker und zur Geschichte der Erde erleben, kreativ gestalten und erlernen können«.

Besonders reiche Orte des Entdeckens und Lernens sind Stätten, die in der Sprache der UNESCO-Welterbekonvention als »combined works of nature and man « beschrieben werden können. Jedes Kulturdenkmal und überhaupt jede vom Menschen angelegte Struktur enthält zwangsläufig Elemente, die die Natur vorgibt, vom Grundstein bis zum Dachgebälk. Die natürlichen Elemente treten in der Wahrnehmung allerdings häufig als überformtes >Materiak in den Hintergrund. Zwischen und unter den Glas- und Stahlkonstruktionen und weiten Asphaltflächen unserer Städte ist die Handschrift der Natur kaum mehr zu lesen.

Die Gartenkunst hingegen arbeitet darauf hin, das >Werk ^ der Natur besonders sichtbar zu machen, und ist, auch wenn ganz erhebliche Eingriffe vorgenommen werden, am Ideal der Natur orientiert.

Was der menschliche Gestalter erschaffen hat, mag »fertig « und als >Werk « abgeschlossen erscheinen, während die natürlichen »kontinuierlichen Prozesse« den Ort über das Handeln des Menschen hinaus weiter formen. Ein Garten ist als »combined work of nature and man « in diesem Sinne nie »fertig «. Ebenso wie Bäume ihr Leben lang weiterwachsen, sich Wildkräuter aussäen, Gewässer zusedimentieren und Hänge aberodieren, ist auch der Eingriff des Menschen in diesem Gefüge ein ständiger Prozess und erfordert ständige Eingriffe in das lebendige, reagierende und emergente Phänomene hervorbringende System.

In den Potsdamer Parklandschaften würde sich ohne beständige Pflegeeingriffe im Park über Jahrhunderte hinweg wieder ein Eichenwald bilden. Potsdam »Poztupini« bedeutet so etwas wie das `Land der Eichen . Die ökologischen und biogeographischen Realitäten haben sich im Ortsnamen niedergeschlagen. Das Toponym »Babelsberg « weist auf einen »Baumeister « der Natur: Im 19. Jahrhundert war der Ort noch als Babers Berg bekannt, was auf das slawische Wort »Bobr « - Biber - zurückgeführt wurde (Berghaus 1854, 364). Der Biber prägte also durch seine Anwesenheit den Ort und tut dies, nach Jahrhunderten der lokalen Ausrottung zurückgekehrt, heute wieder und baut am Menschenwerk um und weiter.

Parks und Gärten können nicht aus ihrem Naturraum herausgenommen und unter Dach gebracht, können nicht eingepackt und "gesichert «, nicht unverändert konserviert werden. Wie viele Welterbestätten und andere historische Flächen und Bauten sind historische Parks in erheblichem Maße vom Klimawandel betroffen (Welling et al. 2015; UNESCO/ UNEP 2016), und die Wirkungen treten deutlich sichtbar in Erscheinung. Sie erweisen sich daher auch als geeignete Lernorte, um dieses Themenfeld zu erschließen (UNESCO/UNEP 2016, 32). Sie können außer »Lernlaboratorien« für die Untersuchung und die Abmilderung von Klimaauswirkungen auch Orte für die Erprobung von »Resilienzstrategien « sein (UNESCO/UNEP 2016, 32). Vielleicht können sie, in Anlehnung an die oben angesprochene Funktion von Schutzgebieten als »Modellregionen für eine nachhaltige Entwicklung« (BfN, 2010; § 25; BNatSchG), sogar in einigen Fällen Modelle für den Umgang mit Klimawandel liefern. 
In Gärten wird in besonderem Maße an den Grenzen des klimatisch Möglichen gearbeitet. Bäume sind zwar aus ihrem natürlichen Konkurrenzgefüge herausgenommen, aber isoliert den klimatischen Bedingungen ausgesetzt. An Standorten, an denen sie in der Natur schon bei nur leicht suboptimalen Bedingungen dem Konkurrenzkampf erlegen wären, können sie im Garten heranwachsen, groß werden und so schließlich die klimatischen Grenzen besonders deutlich machen.

\section{Orts- und objektbasiertes Lernen}

Die in den zitierten Erklärungen und Konventionen betonte Bedeutung der Landschaften als Lernräume konkretisiert sich in lokalen Aktivitäten der Umweltbildung und Denkmalvermittlung und darüber hinausreichenden ortsbezogenen Bildungsansätzen.

Die im angelsächsischen Sprachraum als »Place-based learning « bezeichnete Herangehensweise lässt sich nur unvollständig mit »ortsbasiertem Lernen« übersetzen. »Placebased learning« beschreibt Ansätze, den Ort in seinem gesamten Facettenreichtum und seinen Bedeutungsschichten zu erschließen und für Lernprozesse fruchtbar werden zu lassen. Frühe Wurzeln des »Place-based learning « lassen sich in literarischen Formen der Auseinandersetzung mit Naturräumen insbesondere in Nordamerika finden, wie etwa Aldo Leoplds »Sand County Almnac« (1949) (Warkentin 2011; Kirby 2014).

»Place-based learning« spielt eine besondere Rolle an Colleges, die im ländlichen Raum Nordamerikas Angebote für Native-American-Gemeinschaften erbringen und zu deren Selbstverständnis es gehört, besondere Beziehungen zum Land und dem immateriellen Gehalt des Raumes als Kulturgut und Bildungsinhalt zu pflegen (Semken/Freeman 2008; Kirby 2014). »Place-based learning « findet heute auch in multiethnischen und multikulturellen urbanen Räumen Anwendung, beschränkt sich allerdings hier häufig auf ortsbezogene Arbeit auf dem Schulgelände oder im Schulgebäude (Warkentin 2011), obgleich bereits Thoreau befand: „Sich vorwiegend auf Schreibtisch und Schulhaus zu konzentrieren und gleichzeitig die Landschaft, in der es steht, zu vernachlässigen, ist absurd « (Thoreau 1967, 298).

Eine gründliche, vertiefte und »lernende« Auseinandersetzung mit dem Ort erfordert im Grunde längere Aufenthalte »vor Ort«, dort, wo entdeckt wird, oder zumindest, wie in Ansätzen der »slow pedagogy of place« (Payne/ Wattchow 2009), wiederholtes, regelmäßiges Aufsuchen des Lernortes. Wenn dies etwa über die Jahreszeiten hinweg erfolgt, können die fortwährend neu erwachsenden Phänomene und »Bilder« erlebt werden, etwa, wenn die Szenerie jeweils in ein jahreszeitlich anderes Licht getaucht ist, wenn sich mit dem Wandel der Temperatur, Luftfeuchtigkeit und dem Voranschreiten der Vegetationsperiode eine andere »Landschaft « aus Erd- und Pflanzendüften entfaltet, wenn Flora und Fauna im Jahresgang eine sich stetig ändernde Farbpalette zeigen oder sich die akustische Landschaft zwischen Vogelstimmen und Blätterrauschen stets wandelt.

Ortsbasiertes Lernen oder »Place-based learning « findet wesentliche Parallelen in Ansätzen des objektbasierten Lernens oder »Object-based learning«. Objektbasiertes Ler- 
nen wurzelt in der Museumsdidaktik und bezieht sich meist auf die intensive Auseinandersetzung mit einzelnen ausgewählten Artefakten und die hierdurch ausgelösten und gestützten Lernprozesse (Corbishley 2015; Kador et al. 2017). Objektbasiertes Lernen ist indes auch in den Naturwissenschaften und insbesondere auch mit lebendigen Objekten möglich und hier, wenn auch nicht mit diesem Begriff gefasst, ein selbstverständlicher Bestandteil des Lernens (Robischon 2018).

Auch das objektbasierte Lernen erfordert wiederholte Begegnung, insbesondere, wenn es sich um Objekte im Freiland und Lebewesen handelt. Da ist die Oberfläche eines Gesteins, die sich bei Frost oder Sommerhitze, an einem taufrischen Morgen oder in einer Tropennacht jeweils anders anfühlt. Da ist der Geruch des Wassers - etwa des »Schwarzen Meers « im Park Babelsberg - im Frühjahr oder im Herbst. Pflanzen folgen im Wechsel ihres Erscheinungsbildes den Jahreszeiten, wenn nicht gar Tageszeiten: Im Tagesverlauf ändern sich Objekte wie die Prunkwinde (Ipomoea tricolor), die bei Babelsbergs gotischer Fontäne Hibiskusstämme umrankt: Sie blüht früh morgens in Rosa auf und verfärbt sich im Tagesverlauf in ein helles Blau. Wenn sich die Blüte der Prunkwinde abends schließt, kann man der Nachtkerze (Oenothera biennis) beim Entfalten ihrer schwefelgelben Corolla zuschauen. Zum lebenden und in die lebende Landschaft eingebundenen Objekt gehört eben gerade auch der Zeitbezug.

Selbst wenn Betrachter sich für unbeschränkte Zeit und nicht nur punktuell der Beobachtung widmen könnten, bliebe es unmöglich, den gesamten Bedeutungsgehalt eines Ortes allein mit der eigenen sinnlichen Wahrnehmung zu erfassen. »Erkennen zu wollen, was außerhalb der Erlebniswelt liegt« (Glasersfeld 1992) erfordert Unterstützung durch Lehrende oder zumindest Medien, die erforderliche Informationen liefern oder den Blick auf besonders Aufschlussreiches lenken.

So fallen dem wachen Beobachter zweifellos die mehrstämmigen Eichen des Babelsberger Parks auf, doch nur mit forstlicher oder gartenhistorischer Hintergrundinformation ist zu erschließen, ob sie ihre Form einer früheren Niederwaldwirtschaft oder einer Nesterpflanzung verdanken. Es fällt auf, dass die »Pückler-Pappel«, obgleich auf »freiem Feld « wachsend, eine kleine und außerordentlich hoch angesetzte Krone trägt, doch erfordert das Verständnis des zu vermutenden Eingriffs in die Wachstumsmuster des Baumes ein Wissen um die Bedeutung dieser Pappel als gartenarchitektonisches Element.

Für Lehrende ist es eine besondere Herausforderung, den Kontext der Begegnung so zu gestalten, dass das unmittelbar Erlebte und Beobachtete verstanden werden kann, und Verbindungen zu solchen Fakten, die nicht aus der primären Begegnung ersichtlich sind, hervortreten. Dies ist insbesondere dann wichtig, wenn es sich nur um vergleichsweise kurze, einer Momentaufnahme ähnliche Auseinandersetzungen und Begegnungen mit dem Ort dem Raum und den ihn füllenden und definierenden Objekten - handelt. Es gilt, die sSprache` der Objekte und Phänomene in unsere Sprache zu übersetzen.

Diese Form der Bildungsarbeit vor Ort und am Objekt wird in Anlehnung an das in den Nationalparks der USA entstandene Konzept der heritage interpretation als Natur- und Kulturinterpretation bezeichnet. Doch manchmal schweigen die Dinge. 
Ohne ein Wissen um die "Kartoffelbefehle« Friedrichs des Großen und die (anekdotische) List des Monarchen, die brandenburgischen Bauern dazu zu bewegen, zu essen, was sie nicht kannten, und um ihre historische Bedeutung in einer Zeit der Nahrungsknappheit, ist auch die Bedeutung der Kartoffeln auf der Grabplatte des Königs in Sanssouci schwerlich zu begreifen. Im in die Landschaft eingebrachten Objekt - der schlichten Knolle - bündelt sich die Agrarhistorie zu einer Geschichte, an der jeder, der eine Kartoffel auf das Grab legt, miterzählt.

\section{Narrative Welterschließung}

Geschichten sind vermutlich die älteste Art und Weise, Dinge zu erklären und Bedeutungen darzustellen (Jonassen/Hernandez-Serrano 2002, 66). Sie sind ein Werkzeug »in einer überaus dicht gedrängten und ungeordneten chronologischen Realität «, in einer »konfliktgeladenen und widersprüchlichen Welt« sinnhafte Bedeutungszusammenhänge aufzuzeigen (Cronon 1992). Narrative Strukturen sind unserem Erfahren der Welt inhärent (Cronon 1992) und »wir sehen und erfassen die Welt gedanklich in Geschichten« (Smith 1992, 62). Narrative helfen Menschen, Informationen zu verstehen und in einer Art und Weise, die menschlichen Wahrnehmungsweisen entgegenkommt, zu kommunizieren (Jones/Peterson 2017). Diese Auffassung spiegelt sich bereits in frühen Darstellungen zur nature interpretation wider. In den Worten Enos Mills' ist es Aufgabe der Vermittlung oder interpretation, anhand von »Objekten `Lebensgeschichten $>$ zu erschaffen « (Mills 1923, 186) und »jedem betrachteten Objekt eine interessante Biographie zu geben« $(250)$. Mills $(1923,252)$ betont: »Jede blühende Blume, ebenso wie jeder alte Baum, hat ein abenteuerliches Leben, eine kurze und aufregende Biographie. Die hat auch jedes Stück roten Sandsteins und jeder große Geröllbrocken im Tiefland."

So gilt es, um beispielsweise die Begegnung mit der Siegessäule im Park Babelsberg zu einer Lernerfahrung zu machen, ihre Geschichte zu erzählen, zu der - unter anderem gehört, dass das im 19. Jahrhundert errichtete Monument rund anderthalb Milliarden Jahre (Erd-)Geschichte mit der Granitschale im Berliner Lustgarten teilt. Beide Stücke wurden aus dem einst größten Findling Brandenburgs - einem der beiden Markgrafensteine bei Rauen - hergestellt. Ihre >Biographie< enthält auch eine Eiszeitreise der "großen Geröllbrocken« aus präkambrischem Karlshamn-Granit von der südschwedischen Landschaft Blekinge über das Norddeutsche Tiefland, Jahrzehntausende bevor ein Steinmetz den Meißel ansetzte und mit einem Schlag eine vom Menschen neu geschrieben Geschichte des Objekts begann.

Narrative wachsen um Phänomene und Ereignisse, die sich in Formen und Objekten in die Landschaft einschreiben - oder um Menschen, die beispielsweise das Gesicht der Landschaft planten und als »Naturmaler« die Intention verfolgten, ein bestimmtes Bild zu erstellen, dazu Berge abtrugen und Täler auffüllten, oder, um die Illusion südlicher Vegetation zu erzeugen, am Schloss Babelsberg Schwarzkiefern pflanzten, deren Wipfel gekappt 
wurden, sodass ein schirmartiger, an Pinien der Mittelmeerländer erinnernder Habitus entstand.

Potteiger und Purinton $(1998,11)$ beschreiben als einen wesentlichen Typ von Landschaftsnarrativen »Storytelling landscapes « - »Orte, die gestaltet sind, um eine Geschichte mit explizitem Bezug zu einer Handlung, zu Szenen, Ereignissen, Charakteren etc. zu erzählen«. In einem Garten, dessen Gestaltung ja nie `fertig` ist, bedeutet dies also auch stets ein Neu-Erzählen der Geschichten. Die Nachpflanzung der Pückler-Pappel, die ja immer nur ein Nachbild erzeugen kann, ist auch ein Nacherzählen der Geschichte um diesen Baum.

Manchmal sind diese Geschichten in einem subtilen Subtext »versteckt «, um erst in weiterer Auseinandersetzung gefunden zu werden. Durch Beobachtung allein ist nicht zu erschließen, dass die 20 Linden am Schloss Babelsberg ursprünglich einmal aus dem Garten von Voltaires Potsdamer Wohnhaus verpflanzt worden waren und die jetzt vorhandenen Bäume aus deren Wurzelausschlägen herangezogen wurden.

Zugleich enthält der Ort im Subtext auch viele nur zu erahnende Geschichten, die kein Baumeister oder Gartenarchitekt hier jemals in den Mittelpunkt gerückt hat, und die doch da sind. Es sind zum Beispiel Geschichten um Menschen, die möglicherweise ganz unabsichtlich eine sichtbare, unsichtbare oder immaterielle Spur in der Landschaft hinterließen. $\mathrm{Da}$ ist auch das Narrativ der Menschen, deren Namen wir nicht kennen und deren Beitrag zum Gesamtwerk, beispielsweise als ausführende Hilfsgärtner oder Bauarbeiter, wir nicht in Daten fassen können und an die man bestenfalls in ans Fiktionale reichenden Narrativen erinnern kann.

Viele bedeutungsvolle Objekte und Orte, die dem Uneingeweihten nicht ohne weiteres ins Auge fallen und die keine sichtbaren Spuren historischer Ereignisse tragen, erhalten ihre besondere Bedeutung durch die mit ihnen verbundenen Geschichten (Kaufmann 2009, 42). Oft haben solche Objekte und Orte einen besonderen Wert für Lernaktivitäten, da sie für Lernende eine Überraschung darstellen und Neues, noch Unbekanntes und zur Erkundung Einladendes anbieten können.

Kaufmann $(2009,38)$ schlägt den Begriff »story sites« - »Geschichtenorte« für solche Orte vor, die »[...] als Erinnerungspunkte dienen und gesellschaftlich wertvolle Geschichten ins Gedächtnis rufen: historische Geschichten, die Bezug auf Traditionen geteilter Erinnerung nehmen « und regt an, dass »der Begriff >Storyscape ‘ [also in etwa Geschichtenlandschaft oder narrative Landschaft, Anm. d. Verf.] die Gesamtheit eines ganzen Panoramas solcher Orte bezeichnen« könnte. In der Auseinandersetzung mit dem Ort und dem Erkunden der zugrundeliegenden Geschichten wächst die narrative Landschaft.

\section{Narrative der Umweltveränderung}

In der Entwicklung der Landschaft in »kontinuierlichen Prozessen«, in der noch nicht abgeschlossenen »Biographie« des Ortes und der Objekte kommen stets neue Narrative hinzu. Dies geschieht manchmal durch Großereignisse und im Kielwasser globaler Entwicklun- 
gen: Der Blick vom Babelsberg auf die Glienicker Brücke ist heute mit einer anderen Bedeutung geladen als vor einem halben Jahrhundert, als Deutschland geteilt war.

Ein ganz anderes Narrativ der jüngeren Zeit erzählen auch andere, weitaus kleinere Bruchstücke, die der Teichmuschel (Anodonta cygnea) nämlich, deren olivgrüne und perlmutterne Scherben am Ufer des Wannsees zu finden sind und auf die Anwesenheit von Waschbären hindeuten. Es ist eines von vielen Beispielen von Neozoen und Neophyten, die die Landschaft prägen und verändern. Robinie, Goldrute, Götterbaum und viele andere Arten, die alle einmal als Zierpflanzen zur Gestaltung von Parks und Gärten eingebracht wurden, zeigen ein fortgeschriebenes und sich immer fortscheibendes ökologisches Narrativ der biologischen Globalisierung.

Gerade durch die enge Verbindung des Parkerbes mit den Dynamiken der Natur ist es jederzeit denkbar, dass ein bestimmter Prozess unvorhergesehen Fahrt aufnimmt und dabei womöglich das lange Erhaltene, das »Erbe« eben, in seiner materiellen Form so grundlegend verändert, dass frühere Bedeutungsschichten für uns nicht mehr vollständig lesbar sind - etwa wenn eine Steinskulptur durch sauren Regen bis zur Unkenntlichkeit zerfressen wird oder wenn eine Gehölzart aus dem Gefüge schwindet, weil ein neu eingeschlepptes Insekt oder Krankheitserreger sie auslöscht oder die Umweltbedingungen insgesamt zu ungünstig werden. Dabei können neue Narrative entstehen, die die früheren in den Hintergrund rücken lassen, sie überdecken und palimpsestisch »überschreiben «.

Narrative des Klimawandels werden in einer ersten Näherung zumeist als Rekonstruktion von Klimageschichte verstanden und beschreiben die Entwicklung der geologischen Vergangenheit (Pancost 2017). Eiszeitliche Sande und Findlinge und die Gesellschaften einheimischer Pflanzen können als Zeugen historischer Entwicklungen interpretiert und die bewegte klimatische Vergangenheit so anhand der Ortes und der Objekte kommuniziert werden.

Die in erheblichem Maße vom Klimawandel betroffenen historischen Flächen und Gartenkunstwerke bieten nicht zuletzt, weil häufig Phänomene sichtbar werden, die, indem sie das Werk des Menschen ändern und überformen, auf besondere klimatische Entwicklungen schließen lassen, einzigartige Lernorte zum Thema des Klimawandels,. In einem heißen Sommer, ob nun schon tatsächlich ein Phänomen des Klimawandels oder nicht, mögen die ausgedörrten Rasenflächen und die nach Starkregenfällen ausgeschwemmten Wege einen Anblick bieten, der Vorstellungen von einer Landschaft im Klimawandel entspricht oder diese entstehen lässt. In diesen Bildern zeigt sich, dass in der Betrachtung der Landschaft das Narrativ des Klimawandels bereits eine Rolle spielt und möglicherweise zunehmend die Wahrnehmung des Ortes und seine Bedeutung als Lernraum beeinflussen wird.

\section{Literaturverzeichnis}

Antrop, Marc; Van Eetvelde, Veerle (2017): Landscape Perspectives: The Holistic Nature of Landscape. Dordrecht: Springer.

Berghaus, Heinrich Karl Wilhelm (1854): Landbuch der Mark Brandenburg und des Markgrafthums NiederLausitz in der Mitte des 19. Jahrhunderts: oder geographisch-historisch-statistische Beschreibung der Provinz Brandenburg. Brandenburg: Verlag Adolf Müller. 
Bundesamt für Naturschutz (2010): Großschutzgebiete in Deutschland - Ziele und Handlungserfordernisse Positionspapier des Bundesamtes für Naturschutz. Bonn: Bundesamt für Naturschutz.

Corbishley, Mike (2015): Object lessons in evidence-based learning. In: Darwin-inspired learning. Hg. von Carolyn J. Boulter, Michael J. Reiss und Dawn L. Sanders. Rotterdam: Sense Publishers, 117-129.

Cronon, William (1992): A Place for Stories: Nature, History, and Narrative. In: Journal of American History 78.4, 1347-1376.

Deutsche UNESCO-Kommission (2007): Lübecker Erklärung. Konferenz »UNESCO-Welterbestätten in Europa - Ein Netzwerk für Kulturdialog und Kulturtourismus«. Lübeck, 13.-14. Juni 2007.

http://www.dnk.de/_uploads/media/247_2007_DtUNESCO_LuebeckerErklaerung.pdf

Deutsche UNESCO-Kommission DUK (2006): Resolution zum UNESCO-Welterbe in Deutschland. Hildesheim, 28. bis 29. Juni 2006.

Fløttum, Kjersti; Gjerstad, Øyvind (2017): Narratives in climate change discourse. WIREs Clim Change 8, e429. doi:10.1002/wcc.429 (07.01.2019).

Glasersfeld, Ernst von (1992): Konstruktion der Wirklichkeit und der Begriff der Objektivität. In: Einführung in den Konstruktivismus. München: Piper.

Jonassen, David H., Hernandez-Serrano, Julian (2002): Case-Based Reasoning and Instructional Design Using Stories to Support Problem Solving. In: Educational Technology Research and Development 50.2, 65-77.

Jones, Michael D.; Peterson, Holly (2017): Narrative Persuasion and Storytelling as Climate Communication Strategies. DOI: 10.1093/acrefore/9780190228620.013.384 (07.01.2019).

Kador, Thomas; Chatterjee, Helen; Hannan, Leonie (2017): The materials of life: making meaning through object-based learning in twenty-first century higher education. In: Developing the higher Education Curriculum. Hg. von Brent Carnell und Dilly Fung. London: UCL Press, 60-74.

Kaufman, Ned (2009): Place, Race, and Story: Essays on the Past and Future of Historic Preservation. New York: Routledge.

Kirby, Kent C. (2014): Place in the City: Place-Based Learning in a Large Urban Undergraduate Geoscience Program. In: Journal of Geoscience Education 62.2, 177-186.

Leopold, Aldo (1949): A Sand County Almanac: And Sketches Here and There. Oxford University Press.

Mills, Enos A. (1914): The Story of a Thousand-Year-Pine. Boston/New York: Houghton Mifflin Company.

Mills, Enos A. (1923): The Adventures of a Nature Guide. Garden City. New York: Doubleday, Page \& Company.

Pancost, Richard D. (2017): Climate change narratives. In: Nature Geoscience 10.7, 466-468.

Payne, Phillip G.; Wattchow, Brian (2009): Phenomenological Deconstruction, Slow Pedagogy, and the Corporeal Turn in Wild Environmental/Outdoor Education. In: Canadian Journal of Environmental Education 14, 15-32.

Potteiger, Matthew; Purinton, Jamie (1998): Landscape Narratives: Design Practices for Telling Stories. Chichester, NY: J. Wiley.

Robischon, Marcel (2018, im Druck): Green Brains and Groundsloths. In: The American Biology Teacher.

Semken, Steven; Freeman, Carol B. (2008): Sense of place in the practice and assessment of place-based science teaching. In: Science Education 92.6, 1042-1057.

Smith, Frank (1992): To Think: in language, learning and education. London: Routledge.

Ströter-Bender, Jutta (2011): World Heritage Education: Positionen und Diskurse zur Vermittlung des UNESCOWelterbes. Marburg: Tectum Verlag.

Tang, Chenxi (2008): The Geographic Imagination of Modernity: Geography, Literature, and Philosophy in German Romanticism. Stanford: Stanford University Press.

Thoreau, Henry David (1967): The selected journals of Henry David Thoreau. New York: New American Library. UNESCO/UNEP (2016): World Heritage and Tourism in a Changing Climate. Paris: UNESCO.

UNESCO-Welterbestätten e.V. (2010): UNESCO-Welterbe soll einen Platz in den Lehrplänen bekommen. Jahrestagung der UNESCO-Welterbestätten Deutschland e. V. in Essen. Pressemitteilung.

Warkentin, Traci (2011): Cultivating urban naturalists: Teaching experiential, place-based learning through nature journalizing in Central Park. In: Journal of Geography 110.6, 227-238.

Watson, J. Wreford (1951): The sociological aspects of geography. In: Geography in the twentieth century. Hg. von Griffith Taylor. London: Methuen, 463-499.

Welling, Leigh; Rockman, Marcy; Watson, James; Mackey, Brendan; Potts, Andrew (2015): The role of World Heritage sites in a changing climate. In: World Heritage 77, 4-13. 